\section{MONTE CARLO COMPUTING}

The Monte Carlo Method

The Method of Statistical Trials. By N. P. Buslenko, D. I. Golenko, Yu. A. Shreider, I. M. Sobol' and V. G. Sragovich. Edited by Yu. A. Shreider. Translated from the Russian by G. J. Tee. Translation edited by D. M. Parkyn. (International Sories of Monographs in Pure and Applied Mathematies, Vol. 87.) Pp. xii +381. (London and New York: Pergamon Press, Ltd., 1966.) $80 s$. net.

The Monte Carlo method is a technique for estimating the solution, $x$, of a numerical mathematical problem by means of an artificial sampling experiment. The estimate is a statistic the expected value of which is equal to $x$. Often the mathematical problem itself arises as a problem in probability. If the sampling experiment bears a close relationship to the original problem, then the method is often called the "crude Monte Carlo Method" or the method of simulation.

In 1773 , the naturalist Buffon pointed out that $\pi$ could be estimated by throwing a needle on a striped tablecloth. If the width of each stripe is equal to the length of the needle, the probability that the needle will touch more than one stripe is $2 / \pi$, so this can be estimated by throwing the needle a large number of times. Perhaps the Monte Carlo method should be called "Buffon's method". It has been used many times since, for example by Student in 1908 for discovering the distribution named after him. The method assumed additional importance with the advent of the electronic computer, and it was apparently given its present name during World War II by Fermi, von Neumann and Ulam. There are many problems which are too complicated to solve by conventional methods of calculation, even on an electronic computer, and to which an approximate answer can be obtained on a computer with the help of a suitably constructed sampling experiment. Such an experiment requires the use of random numbers, or, more conveniently, pseudo-random numbers such as the digits in the decimal expansion of $\sqrt{ } 2$.

The present book is, I think, the longest book on the Monte Carlo or Buffon's method, but it is not comprehensive; there is, for example, less overlap than might have been expected with the book by Hammersley and Handscomb.

The chapters doal, in turn, with "Principles", "Inte. grals", "Neutron Physics", "Servicing Processes", "Communication Theory" and "Generation of Random Variables". The applications to communication theory are novel, and arise because of the occurrence of multiple integrals in some problems of detection of signals in noise. Multi-dimensional problems can sometimes be handled by Monte Carlo methods, even in an infinite number of dimensions, when classical methods are impracticablo.

There are naturally a number of errors or important omissions in what is an otherwise useful book. For oxample, on page 6, the heat-conduction equation is mentioned as an example of a problem that can be handled by Monte Carlo methods but "is not connected with the theory of probability". What is overlooked here is that heat is itself based on the same kind of random walking of moleculos that is used in its standard Monte Carlo solution. It is indeed possible that all laws of nature will ultimately be explained by means of the law of large numbers-I believe they will be-but the numerical solution of the mathematical problems will not usually be by direct simulation.

The importance of testing pseudo-random numbers before using them is correctly pointed out but, on page 273 , the very useful "serial test"-in the binary scale it is the most useful test-is incorrectly defined, and a reference is given to a paper in which it was incorrectly used.

There are 282 references.

An English translation was previously published by Elsevier Publishing Company in 1964, but the present version is somewhat enlarged. The editor's name is misspelt on the spine. I. J. GooD

\section{SEQUENTIAL ANALYSIS}

\section{Sequential Methods in Statistics}

By G. Barrie Wetherill. (Methuen's Monographs on Applied Probability and Statistics.) Pp. $x+218$. (London: Methuen and Co., Ltd.; New York: John Wiley and Sons, Inc., I966.) 35s, net.

Dr. Wetherill's book is a useful addition to the literature on sequential analysis. It includes a good many of the results and practical applications of the subject which have appeared since the publication of Wald's book in 1947. The main theoretical results are given, together with the shorter proofs, while the reader is referred to other sources for more lengthy proofs. The remainder of each chapter surveys applications of the procedures proposed, discussing the difficulties involved and modifications which have been tried out.

The first section of the book is devoted to Wald's sequential probability ratio test since it allows general concepts to be illustrated and also some relatively simple results to be obtained. There is then an outline of the principles of decision theory with particular reference to their use for deciding between sequential procedures. The following chapter considers one of the more thorny problems of sequential methods, namely, the question of estimation. The number of observations in a sequential procedure is determined randomly and the argument, which is in essence philosophical, revolves around the question of whether to take this randomness of sample size into account. Dr. Wetherill presents the various opposing points of view clearly and fairly.

Apart from a short account of two-stage sequential plans, the rest of the book is concerned with sequential solutions of problems containing an element of search. These include stochastic approximation methods, procedures for finding maxima on response surfaces, estimation of parameters of quantal response curves and methods for selecting the best of a group of populations.

The book is clearly and concisely written and is amply provided with references. J. W. ThOMPSON

\section{NUMERICAL ANALYSIS}

\section{Theoretical Numerical Analysis}

By Burton Wendroff. Pp. xi +239 . (New York: Academic Press, Inc.; London: Academic Press, Inc. (London), Ltd., 1966.) 888.

THE author's aim is said to be "to present numerical analysis as a legitimate branch of mathematics, doserving attention from mature mathematicians and students alike". It seems to me that the author does not achieve this aim, for several reasons: (i) His idea of "legitimate mathematics" is far too narrow and appears to exclude statistical mathematics and even the highly effective theory of vector spaces-in fact, he confines himself to classical analytical methods, which are often clumsy and sometimes irrelevant. (ii) His subject hardly deserves the title numerical analysis, at least for the first three chap. ters; he concentrates solely on truncation error, and 